Table No. I will at least serve as a rapid method for the standardizing of the stock bottles of natural waters, as the observer's eye becomes trained and more knowledge is obtained of the conditions which influence readings, a more perfect scale may be constructed.

The direct use of the instrument with the construction of a chart for each water, is evidently out of the question where, as in our laboratory, the color must be determined on twenty or thirty samples a day.

A modification of the tintometer has been proposed by $H . R$. Proctor, Yorkshire College, Leeds, England. ${ }^{1}$

This is designed to bring the color patches into juxtaposition as is done in the comparison of two spectra instead of having the separating dark band in the centre. We have not been able to try this instrument, but it promises to be an improvement.

[CONTRIBUTIONS FROM THE CHEMICAL LABORATORY OF THE U. S. DEPARTMENT OF AGRICULTURE, NO. I5.]

\title{
ON THE ESTIMATION OF LEVULOSE IN HONEYS AND OTHER SUBSTANCES.
}

\author{
BY H. W. WILEY.
}

Received September 30,1895 .

$A$ SIMPLE optical method for the estimation of levulose is substances containing that body. The following optical method is one of easy application when the analyst has access to the jacketed observation tube, which will be mentioned below.

The principle of the process rests upon the change in the specific rotatory power of levulose at different temperatures. The change in the optical rotation of other bodies associated with levulose in the conditions of the experiments to be described is so little as to affect the data obtained to a very slight degree. The process, as it has been worked out, is easily applied and gives results which it is believed are better than those obtained by any other method.

The Observation Tube.-The most important adjunct of a mechanical nature in the process is an observation tube which

1 f. Soc. Chem. Ind., I4, 2,1895 . 
will permit of the polarimetric reading of a solution containing levulose at widely separated temperatures. The two temperatures adopted for experimental purposes are zero and $88^{\circ}$. The observation tube which was devised for the purpose is so constructed as to permit of its being surrounded entirely by a temperature control medium, either water or ice. Even the cover glasses of the tube devised are subjected to the temperature of the medium. The whole column of liquid under observation can in this way be brought to the same temperature, thus not only securing more accurate results, but removing the difficulties in the way of reading, produced by the refraction of light in passing through liquid media of different densities. The observation tube employed can be made either of glass or metal. The glass tube changes less in length on changing temperatures, but the metal tube is more sensitive and can be brought with its contents more quickly to a definite temperature. A tube, especially constructed for the purpose and made of silver, is particularly convenient in the observation of solutions which have been inverted by the action of hydrochloric acid. When metal tubes are used the continuous kind is employed, permitting the displacement of one solution by another without the removal of the tube from its position. The length of the tube at ordinary temperature having been determined, correction is made for linear expansion or contraction at the temperature of observation. At low temperatures it is impossible to use an observation tube of ordinary construction on account of the deposition of moisture upon the cover glasses. This difficulty is entirely overcome by attaching to the ends of the observation tube, by means of a threaded screw, a tube made of hard rubber carrying a central axis of perforated brass of the same dimensions as the diameter of the observation tube outside of which are placed fragments of calcium chloride. The end of the tube is covered air-tight with an ordinary cover glass. Being screwed air-tight upon the observation tube the calciun chloride at once absorbs all the moisture within the hard rubber cylinder and thus completely protects the cover glass from the deposition of water. Protected in this way the observation tube can be kept for hours at the temperature of zero, without any possibility of obscuring the 
field of vision from deposited moisture. The construction of the protecting tube, together with the method of its attachments to the observation tube, is shown in the figure.

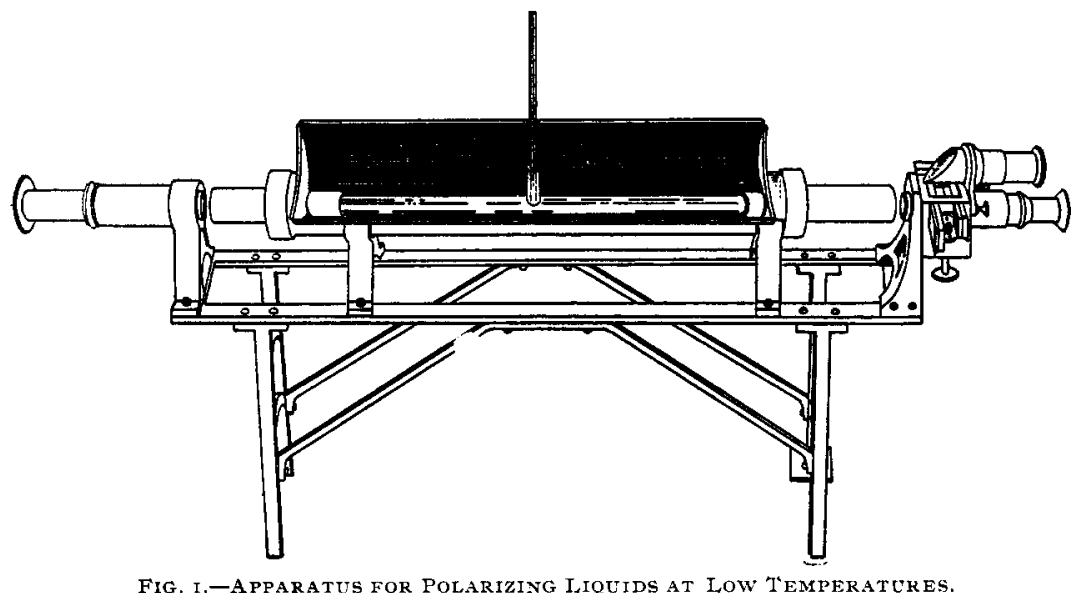

For reading at low temperatures, especially at zero, the observation tube is held in a large metal receptacle coated on the outside with asbestos cloth and having a $V$-shaped slit above. A special stand is made for holding this apparatus to which is also fixed the optical parts of a double compensating Schmidt \& Haensch half shadow polariscope. The construction of the stand and of the holder for the observation tube is shown in the figure, in which, however, the $V$-shaped slit is pushed down to one side in order that the photograph may show the position of the observation tube within. In practice the $V$-shaped slit is kept at the top and the observation tube is entirely surrounded by finely ground ice, an opening in the bottom permitting the water to escape as the ice melts.

The details of the construction of the observation tube are shown in a horizontal section through the center of the observation tube in Fig. 2. In this figure the observation tube, made of glass or metal, is represented by $i$, and the metal jacket, open at the top in the $V$ shape as described, by $k$. The observation tube is closed by the heavy disk $b$, made of non-polarizing glass. This disk is pressed against the end of the observation tube by 
the rubber washer $a$ when the drying system about to be described is screwed on to $k$. The apparatus for keeping the cover glass dry is contained in the hard rubber tube $m$, and consists of a perforated cylinder of brass, $e$, supported at one end by the perforated metal lisk $c$ and at the outer end by the arm $d$. It is closed by a cover glass of non-polarizing glass $s$ and can be screwed on to the system $h$ at $n$. The space $p$ is filled with coarse fragments of caustic soda, or potash, or calcium chloride by removing the cover glass $s$. The perforated disk $c$ prevents any of the fragments from entering the axis of observation.

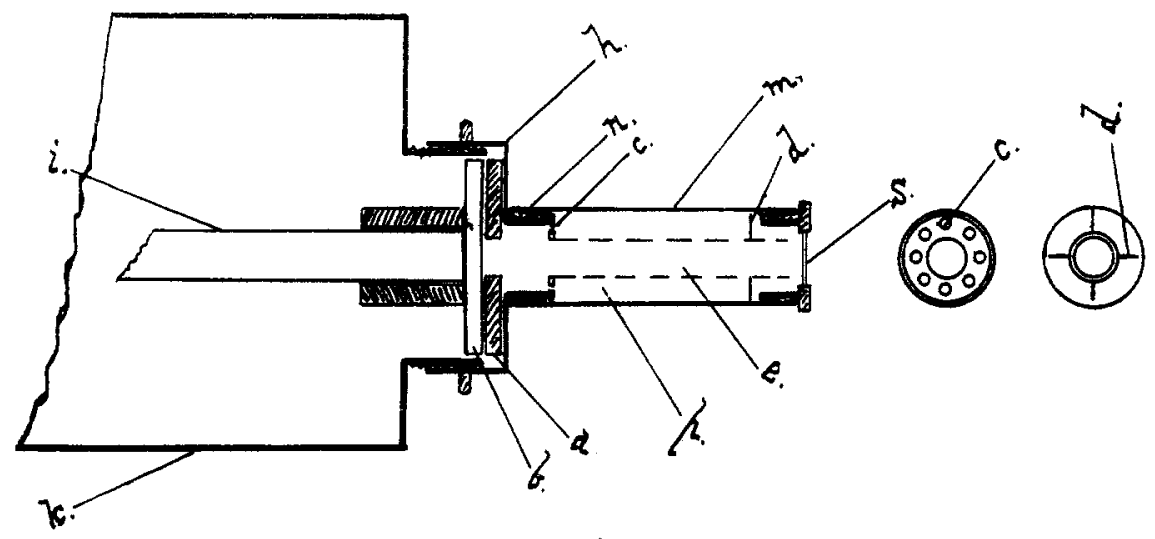

Fig. 2.-Details of Observation Tube.

When the cover glass $s$ is replaced, it just touches the free end of the perforated metal tube preventing any of the fragments of the drying material from falling into the center at the outer end. When this drying tube is placed in position, the contents of the observation tube $i$ can be kept at the temperature of zero for an indefinite time, without the deposition of a particle of moisture either upon the glass $b$ or $s$.

For observation at high temperatures, the apparatus described above may be used or it may be removed from the stand, which is so constructed as to receive a large box covered with as- 
bestos felt an inch thick. The observation tube is held within this box in the same way as in the one just described so that the hot water extends not only the entire length of the tube but also covers the cover glasses. In both cases the cover glasses are made of heavier glass and are much larger in diameter than are the ordinary tubes for polariscopes. The protecting cylinders of hard rubber are not needed at high temperatures, but can be left on without detriment.

The illustration, Fig. 3, shows the arrangement of the apparatus with a silver tube, which can be filled and emptied without removing it, in position.

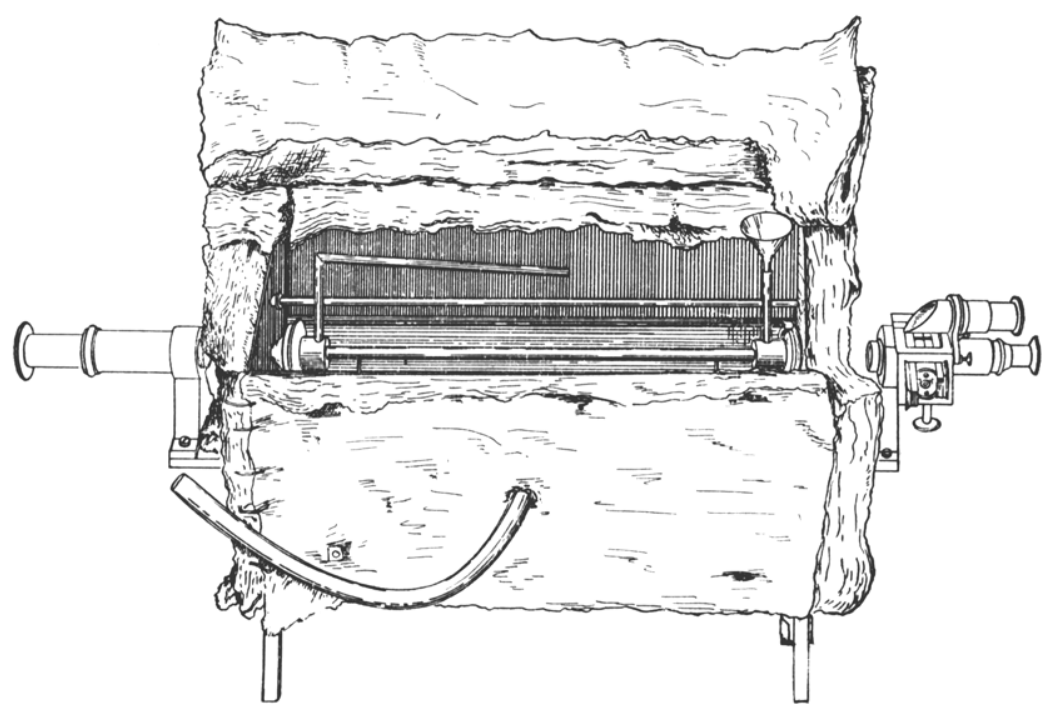

Fig. 3.-Polariscope For L,eVulose at High 'Temperatures.

In practice the water is heated with a jet of steam and an even temperature is secured by a mechanical stirrer, kept slowly in motion. With such a box it is easy to keep a temperature for several hours which will not vary more than half a degree. The temperature for reading the hot solutions is fixed at $88^{\circ}$, this being the one at which a mixture of equal molecules of levulose and dextrose is optically inactive. In every case the sugar solutions are made up to the standard volume at the 
temperatures at which they are to be read, and thus the variations due to expansion or contraction are avoided. When solutions are to be read at a high temperature, they must be made with freshly boiled water so as to avoid the evolution of air bubbles which may otherwise obscure the field of vision.

By means of the apparatus described it is easy for the analyst to make a polarimetric reading at any temperature desired. In all cases the observation tube should be left at least half an hour and sometimes longer, in contact with the temperature control medium before the reading is made. In the case of reading at zero it is also found that it requires several hours for a levulose solution containing other bodies, such as are found in honey, to reach a constant rotatory power. It is therefore necessary, in such cases, to leave the observation tube in contact with finely powclered ice for at least three hours before the reading is made. For ordinary analytical operations, however, this long delay is tunnecessary.

The appearance of the field of vision is usually a pretty fair index of the point of time at which a constant temperature is established throughout all parts of the system. Any variation in temperature produces a distortion of the field of vision, while a constant fixed temperature will disclose the field of vision in its true shape and distinctness of outline.

The chief points of novelty in the apparatus described are, first, the immersion of the whole of the observation tube together with the cover glasses in the temperature medium used; and second, the protection of the external surfaces of the cover glasses from the deposition of moisture at low temperatures.

PRINCIPLES OF THE CALCULATION.

If 26.048 grams of pure sucrose be dissolved in water and the volume made up to IOo $\mathrm{cc}$. it will produce an angular rotation of $34.68^{\circ}$ when examined in a $200 \mathrm{~mm}$. tube with polarized sodium monochromatic light. Upon the cane sugar scale of an accurately graduated Schmidt and Haensch shadow instrument the reading will be Ioo divisions, corresponding to Ioo per cent. of pure sucrose. 
In the complete inversion of the cane sugar the reaction which takes place is represented by the following formula :

$$
\mathrm{C}_{12} \mathrm{H}_{22} \mathrm{O}_{11}+\mathrm{H}_{42} \mathrm{O}=\mathrm{C}_{6} \overline{\mathrm{H}}_{12} \mathrm{O}_{6}+\mathrm{C}_{0} \mathrm{H}_{12} \mathrm{O}_{6} \text {. }
$$

The minus and plus signs indicate that the resulting invert sugar is a mixture of equal parts of levulose ( $l$ fructose) and dextrose ( $d$ glucose). We are not concerned here with the fact that a complete inversion of cane sugar is a matter of great difficulty nor with the danger which is always experienced of destroying a part of one of the products of inversion. They are matters which may cause a variation in the analytical data afterward, but do not affect the principles on which the process is based.

In the inversion of 26.048 grams of cane sugar there are therefore produced I 3.7 I grams of levulose and 13.7 I grams of dextrose, or in all 27.42 grams of the mixed sugars.

The angular rotation which would be produced with sodium light, by 13.7 I grams of dextrose in a volume of roo cc. and through a column $200 \mathrm{~mm}$. in length is, $14.53^{\circ}$, equivalent to $4 \mathrm{r} .89$ divisions of the cane sugar scale. The specific rotatory power of dextrose solution of the density given is almost exactly 53 , and this number is used in the calculations.

In a mixture of the two sugars under the conditions mentioned and at a temperature of $0^{\circ}$, the angular rotation observed is - $15.15^{\circ}$ equivalent to 43.37 divisions of the cane sugar scale.

The + rotation due to the dextrose present is $14.53^{\circ}$. Therefore, the total negative rotation due to levulose at $0^{\circ}$ is $15.15+$ $\mathrm{I} 4.53=29.68^{\circ}$. The specific rotatory power, therefore, of levulose at $0^{\circ}$ and in the degree of concentration noted is readily calculated from the formula

$$
-(\alpha)_{\mathrm{D}}^{\circ}=-\frac{29.68 \times 100}{2 \times 13.7 \mathrm{I}}=108.24 .
$$

Since at $88^{\circ}$ the mixture of levulose and dextrose is neutral to polarized light, it follows that at that temperature the specific rotatory power of levulose is equal to that of dextrose, viz. $: 53^{\circ}$.

$$
-(\alpha){ }_{\mathrm{D}}^{88^{\circ}}=-53^{\circ} \text {. }
$$


The total variation in the specific rotatory power of levulose, between zero and $88^{\circ}$, is $108.24-53=55.24^{\circ}$. The variation for each degree of temperature, therefore, of the specific rotatory power of levulose is equal to 55.24 divided by 88 , which is equal to $0.628^{\circ}$. From these data it is easy to calculate the specific rotatory power of levulose for any given temperature. For instance, let it be required to determine the specific rotatory power of levulose at a temperature of $20^{\circ}$. It will be found equal to $108.24-$ $0.628 \times 20=95.68^{\circ}$.

In these calculations the influence of the presence of hydrochloric acid upon the rotatory power of the levulose is neglected.

Since the variation in angular rotation in the mixture at different temperatures is due almost wholly to the change in this property of the levulose, it follows that the variation for each degree of temperature and each per cent. of levulose can be calculated. Careful experiments have shown that the variation in the rotatory power of levulose between $0^{\circ}$ and $88^{\circ}$ is represented by a straight line. For I 3.7 grams per $100 \mathrm{cc}$. the variation for each degree of temperature is equal to $43.37 \div 88=0.49$ division on the cane sugar scale or $15.15 \div 88=0.1722^{\circ}$ angular measure. If 13.7 I grams of levulose in $100 \mathrm{cc}$. produce the deviations mentioned for each degree of temperature, one gram would give the deviation obtained by the following calculations :

For the cane sugar scale,

And tor angular rotation,

$$
0.49 \div 13.71=0.0357^{\circ}
$$

$$
0.1722 \div 13.71=0.01256^{\circ}
$$

The above data afford a simple formula for calculating the percentage of levulose present from the variation observed in polarizing a solution containing levulose, provided that the quantity of levulose present is approximately fourteen grams per IOO cc.

Suppose in a given case the difference of reading between a solution containing an unknown quantity of levulose at $0^{\circ}$ and $88^{\circ}$ is equal to thirty divisions of the cane sugar scale. What weight of levulose is present? We have already seen that one gram in roo cc. produces a variation of 0.0357 division for $\mathrm{I}^{\circ}$. For $88^{\circ}$ this would amount to 3.1416 divisions. The total 
weight of levulose present is therefore $30 \div 3.14 \mathrm{I} 6=9.549$ grams. In the case given 26.048 grams of honey were taken for the examination. The percentage of levulose was therefore $9.549 \div 26.048=36.66$ per cent.

If it be inconvenient to determine the polarimetric observations at temperatures so widely separated as $0^{\circ}$ and $88^{\circ}$ the interval may be made less. In the above case if the readings had been made at $20^{\circ}$ and $70^{\circ}$ the total variation would have been only 50/88 of the one given, viz.: I 7.05 divisions of the cane sugar scale. The calculation would then have proceeded as follows :

$$
0.0357 \times 50=1.785 \text {. }
$$

Then $17.05 \div 1.785=9.552$ grams of levulose, from which the actual percentage of levulose can be calculated as above.

For honeys the operation is to be conducted as follows:

Since honeys contain approximately twenty per cent. of water and in the dry substance have approximately forty-five per cent. of levulose, about 38.50 grams of the honey should be taken to get approximately 13.8 gram of levulose.

In the actual determination the calculations may be based on the factors above noted, but without respect to the degree of concentration. If only half the quantity of dextrose noted be present its specific rotatory power is only reduced to $52.75^{\circ}$ and this will make but little difference in the results. In the case of honey only 13.024 grams of the sample are used in the examination, half the normal weight for the Ventzke sugar scale. The error however due to difference in concentration is quite compensated for by the ease of clarification and manipulation. Alumina cream alone is used in the clarification, thus avoiding the danger of disturbing the rotatory power of the solution due to the presence of an excess of lead acetate.

An interesting fact is observed in cooling solutions of honey to $0^{\circ}$. The maximum left-handed rotation is not reached as soon as the temperature reaches $0^{\circ}$, but only after it has been kept at that temperature for two or three hours. The line representing the changes in rotatory power, in solutions of honey between $0^{\circ}$ and $88^{\circ}$, is practically straight but from $10^{\circ}$ to $0^{\circ}$, if measured by the readings taken without delay, it is decidedly 
curved; the reading being less at first than it really is afterwards. After three hours the $o^{\circ}$ becomes sensibly constant and then the whole line is nearly straight but still with a slight deficiency in the reading at the $0^{\circ}$. For this reason the computations should be based on readings between $0^{\circ}$ and $88^{\circ}$ rather than on a number covering the whole range of temperature. Nevertheless, if the solution be kept at $0^{\circ}$ for three hours before the final reading is taken no error of any practical magnitude can be introduced.

The calculations given above for the cane sugar scale can also be made in an exactly similar manner for angular rotation. The angular variation produced by one gram of levulose for $1^{\circ}$ of temperature is $0.01256^{\circ}$. For $88^{\circ}$ this would become $1.10528^{\circ}$. Suppose the total observed angular deviation in a given case between $0^{\circ}$ and $88^{\circ}$ to be $10.404^{\circ}$. Then the weight of levulose present is $104.04 \div \mathrm{I} .10529=9.4 \mathrm{I} 3$ grams.

In the case mentioned 26.048 grams of honey were taken for the examination. The percentage of levulose present therefore was $9.4 \mathrm{I} 3 \div 26.048=36 . \mathrm{I} 3$.

GENERAL FORMULA FOR THE CALCULATION OF PERCENTAGE OF LLEVULOSE.

Let $K=$ deviation in divisions of cane sugar scale or in angular rotation produced by one gram of levulose for $I^{\circ}$ temperature.

Let $T$ and $t^{\prime}=$ temperatures at which observations are made. Let $R=$ observed deviation in rotation.

Let $W=$ weight of levulose obtained.

Let $L=$ per cent. of levulose required.

Then

$$
L=\frac{R}{K\left(T-t^{\prime}\right)} \div W
$$

In most genuine honeys the value of $R$ between $0^{\circ}$ and $88^{\circ}$ is approximately thirty divisions of the cane sugar scale or $10^{\circ}$ angular measure for $26.048 \mathrm{grams}$ in $100 \mathrm{cc}$. read in a $200 \mathrm{~mm}$. tube, or for 13.024 grams in $100 \mathrm{cc}$. read in a $400 \mathrm{~mm}$, tube.

The method of analysis outlined above has been applied in the examination of a large number of honeys with most satisfactory results. It can also be applied with equal facility to other substances containing levulose. 